\title{
LA UNIDAD DIDÁCTICA (INTEGRADA): DOCUMENTO PROGRAMADOR PARA LA INNOVACIÓN-INDAGACIÓN EDUCATIVA(S) EN/DESDE UNA PERSPECTIVA INCLUSIVA. UN ESTUDIO DE CASO
}

\author{
The Didactic Unit (integrated): Programming Document \\ for Educational Innovation-inquiry (s) in / from \\ an Inclusive Perspective. A Case Study
}

Blas CAMPOS BARRIONUEVO

Recibido: 30/06/2020; Aceptado: 15/07/2020; Publicado: 30/12/2020

Ref. Bibl. BLAS CAMPOS BARRIONUEVO. La unidad didáctica (integrada): documento programador para la innovación-indagación educativa(s) en/desde una perspectiva inclusiva. Un estudio de caso. Enseñanza \& Teaching, 1-2020, 147-175.

RESUMEN: Mediante el presente trabajo realizamos un recorrido teórico y procedimental (investigador) de la necesidad (de actualizar y regenerar) el ejercicio programador docente, en su proceso de contextualización curricular, sobre todo en la educación secundaria obligatoria (en adelante, ESO), como una experiencia llevada a cabo en un instituto de enseñanza secundaria (a modo de estudio caso único); todo ello, para dar respuesta a las demandas del profesorado de dicho centro, amén de una progresiva cualificación de los procesos educativos a realizar en dicho centro escolar. El proceso investigador, con una metodología cualitativa, utilizando como técnica-base tanto el cuestionario abierto y cerrado como, sobre todo, los grupos de discusión (en base a seis categorías), se ha realizado en dos fases sucesivas, yendo de la necesidad del ejercicio programador general a otro más particular e integrador -interdisciplinariedad-. A través de las conclusiones y discusiones se ha evidenciado la transferencia de dicho ejercicio programador a nivel docente, tanto en la mejora de los métodos de enseñanza cuanto en la mejora de los resultados escolares del alumnado, en general. Finalmente, y a modo de modelo-base, se ha establecido un 
diseño de programación por unidades didácticas para todo el profesorado de dicho centro, sin menoscabar su transferencia a otros centros y docentes de dicha zona u otras (zonas) educativas. Hay que destacar, por otra parte, la riqueza de dicha experiencia en todos sus ámbitos, activando la necesidad de investigar (desde la acción) toda acción escolar y, en particular, aquellas que tienen por objeto el diseño curricular de los procesos de enseñanza.

Palabras clave: currículo; interdisciplinariedad; currículo integrado; programación; unidad didáctica; grupo-aula; educando; elemento curricular.

SUMMARY: Through this work, we carry out a theoretical and procedural tour (researcher) of the need (to update and regenerate) the teaching programmer exercise, in its process of curricular contextualization, especially in compulsory secondary education (hereinafter, ESO), as a experience carried out in a secondary school (as a single case study); all this, to respond to the demands of the teachers of said center, in addition to a progressive qualification of the educational processes to be carried out in said school. The research process, with a qualitative methodology, using the open and closed questionnaire as a base technique, and, above all, the discussion groups (based on six categories), has been carried out in two successive phases, going from the necessity of the general programmer exercise, to a more particular and integrating one -interdisciplinarity-. Through the conclusions and discussions, the transfer of said programming exercise to the teaching level has been evidenced, both in the improvement of teaching methods and in the improvement of the student's school results, in general. Finally, and as a base model, a programming design has been established by didactic units for all the teachers of said center, without undermining its transfer to other centers and teachers in said zone or other educational zones. On the other hand, it is necessary to highlight the richness of this experience in all its fields, activating the need to investigate (from the action) all school actions and, in particular, those that have as their object the curricular design of the teaching processes.

Key words: curriculum; interdisciplinarity; integrated curriculum; programming; didactic unit; group-classroom; student; curricular element.

\section{INTRODUCCIÓN/JUSTIFICACIÓN}

Los institutos de educación secundaria (en adelante, IES) constituyen una verdadero reto de formación y actualización permanente(s) de su profesorado, dada la complejidad curricular y convivencial inherente a los mismos, sobre todo en el periodo de la educación secundaria obligatoria (en adelante, ESO) -12/16 años-, dada la significatividad evolutiva de dicho alumnado [adolescencia y sus vicisitudes tanto en el contexto familiar como escolar (García, 2019)], la diversidad curricular, la inclusión socioeducativa, la problemática convivencial, etc. Es por ello que debe haber un proceso de actualización, acomodación y regeneración de las didácticas 
empleadas, bien a nivel general, bien de forma específica, en un intento permanente de mejora de los procesos de enseñanza y, por ende, de los rendimientos escolares de su alumnado, en general, y de los educandos que cursan la ESO, en particular. Ante dicha situación, nos hemos planteado y elaborado una investigación, a modo de estudio de caso único -un IES-, con la finalidad de mejorar el proceso programador de su profesorado, tanto de forma individual-contextualización progresiva de la praxis docente- como grupal, bien a nivel de los departamentos didácticos (programación vertical), bien a nivel de los equipos educativos (programación horizontal), sin menoscabar la generalización de los resultados obtenidos a otros IES de una misma zona educativa (o distinta) a través de la actualización y elaboración conjunta de sus proyectos educativos en dichos espacios escolares.

Por otra parte, (en) los IES, donde confluyen y conviven docentes de distinta naturaleza, en cuanto a formación, titulación de ingreso, preparación y formación pedagógica(s); en definitiva, pertenecientes a distintos cuerpos docentes, ..., amén de la diversidad de su alumnado en el sentido amplio del término como ya se ha apuntado supra, conforman a dicho profesorado en un agente fundamental para reordenar y actualizar su proceso programador; en síntesis, su praxis docente.

La inclusión escolar es una dimensión nuclear de la calidad educativa que debe ser abordada desde el propio ejercicio programador, atendiendo a la diversidad del alumnado en las dimensiones de tipo social, personal, familiar y cognitivo, fundamentalmente.

Mediante esta investigación, y en una visión interdisciplinar (currículo integrado) pretendemos vertebrar la atención a la diversidad del alumnado en sus aptitudes/actitudes hacia los aprendizajes escolares desde dicho ejercicio planificador en la implementación de los actos didácticos en el primer ciclo de la ESO $\left(1 .^{\circ}, 2 .^{\circ}\right.$ y 3.er niveles), sobre el alumnado que presente una mayor dificultad en la adquisición de sus aprendizajes, así como (una) mayor problemática en su integración convivencial en dichos centros escolares.

Obviamente, esta realidad es diferencial de unos centros a otros, así como de unas zonas educativas a otras; consecuentemente, se hace necesario establecer un centro de referencia como estudio de caso, en un intento de investigar, indagar, innovar y, en última instancia, responder a las demandas didácticas del profesorado que lo constituye, máxime ante la inquietud global a nivel docente en relación al elemento curricular, competencias clave; en concreto, cómo programarlas y (cómo) medirlas, entre otros elementos del currículo a considerar.

El proceso de generalización consistente en estudiar diferentes centros docentes y compararlos entre sí constituye un trabajo que desborda los límites del presente, dejando dicha cuestión para un proceso de investigación más amplio. 


\section{MARCO TEÓRICO/FUNDAMENTACIÓN}

\subsection{La Unidad Didáctica (en adelante, ud): diseño y desarrollo a nivel metodológico ${ }^{1}$}

Las uu. dd. tienen una gran importancia como instrumento de trabajo dentro del ejercicio programador docente, de cara a la contextualización curricular (y progresiva) a nivel de cada grupo-aula. Una UD es una unidad de trabajo operativa, versátil e integrada, al contener todos los elementos curriculares que vertebran la praxis docente, en torno a una línea-eje que la estructura. El término UD no es nuevo, ya que el plan Morrison de los años 30 del siglo xx, con un mayor apogeo en la década de los 60, y conocido también como plan de las unidades didácticas, se centraba, a modo de síntesis, en los siguientes presupuestos: a) alta funcionalidad en las interacciones del docente con su alumnado; b) activación del docente en la organización de su trabajo, así como en la consignación de las actividades-tareas a desarrollar en los grupos-aula; y c) dicho plan obedecía a las fases de desarrollo didáctico: exploración, presentación, asimilación, organización y recitación. En definitiva, el término unidad didáctica, importado de Estados Unidos, nació como una reacción al asignaturismo.

El término UD no pertenece en exclusiva a una escuela o enfoque; solo cobra sentido y coherencia en su contexto, correspondiéndose con orientaciones metodológicas y modelos de enseñanza-aprendizaje singulares. Por otra parte, tampoco hay una única metodología para el diseño y elaboración de las unidades didácticas.

Seleccionamos algunas definiciones para, a continuación, proponer una (concreta) desde un enfoque más comunicativo-interactivo, en una visión más funcional y (transferencial) para nuestros propósitos educativos.

Gimeno (1988) afirma:

Esa fórmula supone el currículum en unidades con sentido en sí mismas, que pueden añadirse a otras para cubrir un área o un objetivo amplio y a largo plazo. La secuencia de actividades ofrece alternativas diversas a concretar de acuerdo con la propia entidad del contenido, las opciones pedagógicas y las ventajas de organización del currículum en periodos o niveles educativos. (p. 361)

Escamilla (1993) afirma: "Una forma de planificar el proceso de enseñanzaaprendizaje alrededor de un elemento de contenido que se convierte en el eje integrador del proceso, aportándole consistencia y significado» (p. 39).

Desde nuestra concepción la UD debe concebirse como un plan abierto, flexible, regenerable y dinámico a lo largo del proceso de enseñanza-aprendizaje que busca la consecución de objetivos y adquisición de competencias (como saber hacer funcional), en un determinado periodo de tiempo, basado en tareas globales,

1. En este apartado, explicitamos una visión del diseño y desarrollo del currículo, desde la perspectiva de un sistema metodológico integrado. 
LA UNIDAD DIDÁCTICA (INTEGRADA): DOCUMENTO PROGRAMADOR PARA

LA INNOVACIÓN-INDAGACIÓN EDUCATIVA(S) EN/DESDE UNA PERSPECTIVA INCLUSIVA. UN ESTUDIO DE CASO mediante centros de interés-núcleos temáticos de acción formativa, permitiendo la comprensión, aprendizaje y reflexión del área/materia en cuestión.

\subsection{Modelo-base de programación didáctica en el aula: diseño}

La planificación de un currículo en diversos niveles de contextualización/ concreción curricular, establecido de una forma flexible y abierta, exige del profesorado una nueva visión del proceso programador en el aula (contextualización curricular), a nivel individual y colectivo, como equipos docentes.

Por otra parte, la determinación desde la Administración educativa en la Comunidad Autónoma de Andalucía, de desarrollar y complementar el currículo establecido en el ámbito de la autonomía de los centros educativos [ex art. 8.1, del Decreto 111/2016, de 14 de junio, por el que se establece la ordenación y el currículo de la ESO en la Comunidad Autónoma de Andalucia, en concordancia con el art. 7.2 del Real Decreto 1105/2014, de 26 de diciembre, por el que se establece el currículo básico de la ESO y del bachillerato; ver al respecto, bibliografía infra, exige, además, una acotación funcional a las necesidades del alumnado, bien de forma individual, bien a nivel del grupo-aula, redundando todo ello, en un ejercicio programador docente como, por otra parte, se establece en la LOE-TC², ex art. 91, 1, a), al precisarse como una de las funciones del profesorado, la programación y la enseñanza de las áreas, materias o módulos que se tengan encomendados. En mayor o menor medida, esta temática ha derivado en una determinada inquietud por parte del profesorado de educación secundaria, preferentemente del Cuerpo de Profesores de Enseñanza Secundaria [datos procedentes del cuestionario aplicado al profesorado del centro objeto de estudio], así como también en la demanda por parte de ellos de aprender, ser asesorados y trabajar en equipo este ámbito de trabajo, con la finalidad de mejorar su propia práctica docente (aspecto problema, opción 1 a nivel investigador, véanse objetivos de la investigación, infra).

Más específicamente, y a modo de ilustración, sintetizamos lo expuesto en la Figura 1, infra:

2. Ley Orgánica 2/2006, de 3 de mayo, de Educación (2006). Texto Consolidado, tras su (nueva) redacción, dada por la Ley Orgánica 8/2013, de 9 de diciembre, para la Mejora de la Calidad Educativa (LOMCE). 


\section{FIGURA 1}

Ideograma en el diseño de una UD. Cualificación de la praxis docente

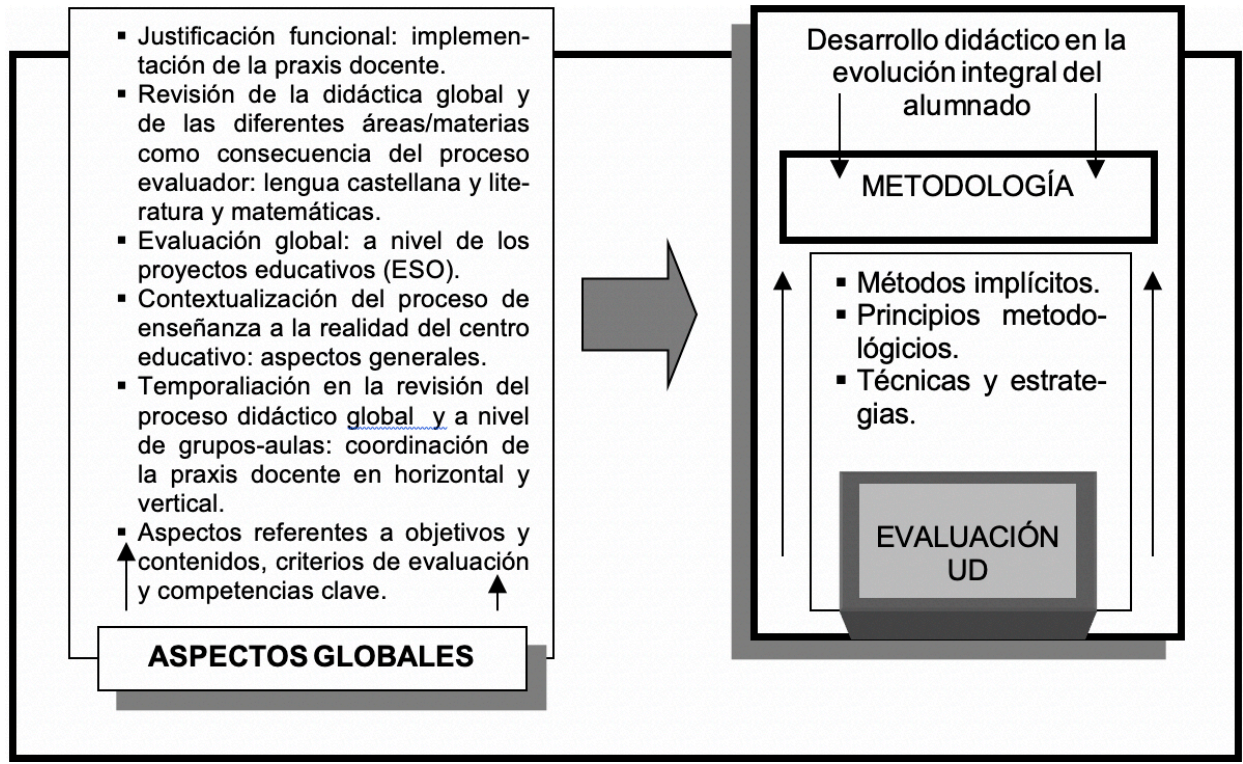

Elaboración personal.

\section{OBjETIVOS DE LA INVESTIGACIÓN}

Vistas las premisas de referencia anteriores, hemos realizado una "misma | única» investigación, con dos niveles de incidencia didáctico-metodológica, considerando ambos referidos a un estudio de caso -un instituto de educación secundaria con 60 docentes-, sin establecer hasta la actualidad ninguna comparación, en relación al mismo ámbito de estudio, con otros centros docentes de la misma tipología. En dicha investigación ha habido una implicación alta y aceptable de los docentes de dicho centro que, por otra parte, han sido los agentes demandantes de dicha experiencia, previa estimulación e incentivación por parte de la dirección y jefatura de estudios de aquel, ante las inquietudes curriculares de aquel. Además, el alumnado (grupos de discusión) del 1.er ciclo de ESO se ha mostrado muy receptivo, ante la problemática lectora detectada como consecuencia de las pruebas estandarizadas y (no homologadas - por su profesorado-) realizadas en los últimos cursos escolares en dicho centro.

En síntesis, se ha establecido la investigación, de acuerdo a la siguiente Tabla 1, infra: 
LA UNIDAD DIDÁCTICA (INTEGRADA): DOCUMENTO PROGRAMADOR PARA

LA INNOVACIÓN-INDAGACIÓN EDUCATIVA(S) EN/DESDE UNA PERSPECTIVA INCLUSIVA. UN ESTUDIO DE CASO

TABLA 1

Investigación-innovación metodológica

\begin{tabular}{|c|c|}
\hline $\begin{array}{c}\text { Investigación } \\
\text { Objetivo 1 }\end{array}$ & $\begin{array}{c}\text { Generar modelos base de programación didáctica } \\
\text { contextualizados a los grupos-aula }\end{array}$ \\
\hline \multirow{2}{*}{$\begin{array}{c}\text { Desarrollar la interdisciplinariedad en el primer ciclo de la ESO, } \\
\text { Objetivo } 2\end{array}$} & $\begin{array}{c}\text { en aras de potenciar el aprendizaje y la evaluación de las áreas } \\
\text { instrumentales [adquisición y potenciación de las competencias } \\
\text { clave en lengua y matemáticas: ámbito lector, como eje globalizador- } \\
\text { integrador de las competencias clave (comunicativo)-lingüística } \\
\text { y matemática [ver RD } 1105 / 2014 \text {, de 26 de diciembre, por el que } \\
\text { se establece el currículo básico de la ESO: anexo I, pp. 357/364] }\end{array}$ \\
\hline
\end{tabular}

Elaboración personal.

\section{Metodología}

Nuestra investigación se ha centrado en el primer ciclo de secundaria $\left(1 .^{\circ}, 2^{\circ}{ }^{\circ}\right.$ y $3 .^{\circ}$ ), dejando para cursos posteriores su transferencia plena a otros niveles superiores, tanto en ESO como en Bachillerato y Formación Profesional Inicial en los ciclos impartidos en el centro objeto de estudio e investigación. Por otra parte, ha sido en este primer ciclo donde se ha demandado una mayor inquietud didáctica en los últimos años, aunque ello no implica que en niveles superiores también la haya habido con un interés más reducido, en relación a los aspectos de programación y seguimiento de las clases en las diferentes materias/asignaturas, en general, y, de forma más particular, en las instrumentales de lengua castellana y literatura y matemáticas, así como en sus correspondientes programas de refuerzo, en relación al alumnado necesitado de ellos y, lógicamente, en el marco normativo vigente para dicha tarea.

Hay que destacar que la asignación en los niveles iniciales de la EsO de maestros y profesores de enseñanza secundaria ha facilitado el trabajo colaborativo y recíproco en dicho cometido.

Más concretamente, la secuencia metodológica (a nivel investigador) ha sido la siguiente:

Delimitación del problema de investigación: inquietud y avidez hacia el aprendizaje programador en la mayoría de los docentes del primer ciclo de la ESO, en un proceso de contextualización curricular.

Modelo de innovación docente-institucional: documental, biográfico-narrativo y etnográfico.

Modelo didáctico a innovar: en primer lugar, se procedió a establecer varias reuniones iniciales y "con carácter de pilotaje», con un número de docentes reducido (voluntarios), con la finalidad de sensibilizarlos, asesorarlos desde el departamento de orientación/jefatura de estudios e incentivarlos 
a generar un cambio progresivo, en la manera de ver su ejercicio programador. En una segunda fase, de una forma comunicativa-interactiva se procedió a irradiar dicha estimulación a los colegas de los departamentos didácticos afectados y, en último lugar, se estableció una reunión general con todo el claustro (generando un grupo de trabajo) bajo la coordinación del orientador/jefe de estudios para iniciar un cambio en la concepción curricular de todo el claustro. Obviamente, la situación ha sido diferencial entre los docentes, lo cual ha determinado propiciar un coasesoramiento con el propio orientador/jefe de estudios. Después y durante todo este proceso se ha generado un modelo de colaboración y comunicación-interacción con una cultura colaborativa que, por otra parte, se ha enriquecido con la incorporación de nuevos docentes («más innovadores») en los dos últimos cursos.

Metodología de investigación-innovación: entrevistas individuales y grupales; revisión de documentos del profesorado; entrevistas biográficas; grupos de discusión, observación participante, etc.

Contexto de investigación: realidad implícita en el aula y, por extensión, departamentos didácticos; se implicaron (inicialmente) diez docentes, de diferentes materias, siendo mayoritarios en lengua castellana y literatura y matemáticas.

Proceso de indagación: actualización y revisión del proceso programador durante dos cursos escolares, con la pretensión de extrapolarlo al resto de docentes, desde la perspectiva de asesores como colegas, durante el siguiente quinquenio.

Método de investigación: la investigación-acción, reformando procesos educativos como una reflexión de la propia práctica, a nivel individual y grupal como docentes.

Recogida de datos: se han elaborado fichas y documentos de todo tipo, modelos provisionales para programar e interpretar las programaciones didácticas de departamento, etc.

\section{ANÁLISIS DE RESULTADOS}

El análisis de contenido se ha llevado a cabo mediante un proceso de triangulación y elección selectiva de las frases-voces claves de las respuestas a las cuestiones abiertas del cuestionario y entrevistas (realizadas), así como (de) los grupos de discusión, sin menoscabar aquellas cuestiones de tipo más cerrado; todo ello, en un intento de avanzar en la temática de estudio y corroborar la necesidad de una mayor activación y sensibilización en el ejercicio programador docente en toda su extensión y, por ende, determinar su implicación en la mejora de la praxis docente. 
LA UNIDAD DIDÁCTICA (INTEGRADA): DOCUMENTO PROGRAMADOR PARA

LA INNOVACIÓN-INDAGACIÓN EDUCATIVA(S) EN/DESDE UNA PERSPECTIVA INCLUSIVA. UN ESTUDIO DE CASO

TABLA 2

¿Qué percepción se tiene del ejercicio programador docente en el centro?

\begin{tabular}{|c|c|}
\hline PROFESORADO & EQUIPO DIRECTIVO \\
\hline $\begin{array}{l}\text { - En general, es una situación automá- } \\
\text { tica, aunque falta reflexionar y ahon- } \\
\text { dar en esta cuestión con el pertinente } \\
\text { asesoramiento, dada la actual estructura } \\
\text { curricular. } \\
\text { - Nos gustaría concretar y avanzar en este } \\
\text { campo, sobre todo para llegar mejor al } \\
\text { alumnado. } \\
\text { - Es una cuestión que necesita más tiempo } \\
\text { de reflexión y trabajo en equipo. } \\
\text { - Es un tema que se debe ver en cada } \\
\text { departamento didáctico para su posterior } \\
\text { traslado a los grupos de alumnos. }\end{array}$ & $\begin{array}{l}\text { - Hace falta reflexionar sobre este tema } \\
\text { y sensibilizar a todo el profesorado } \\
\text { sobre la importancia de programar con } \\
\text { adecuación. } \\
\text { - Hay que desarrollar una nueva cultura } \\
\text { en este campo curricular con el apoyo } \\
\text { de la Inspección educativa, centros de } \\
\text { formación de profesorado... }\end{array}$ \\
\hline
\end{tabular}

\section{Grupo de discusión}

Los participantes en el grupo: equipo directivo, orientador escolar, profesorado de los departamentos de lengua castellana y literatura y matemáticas, han manifestado:

- Es complicado programar, hay muchas tareas a realizar en el centro y no tenemos modelos de referencia operativos.

- Con un espacio concreto y con una planificación y apoyo del centro de profesorado (a medio plazo) se podría avanzar en este campo curricular (orientador escolar).

- Enseñamos bien, pero necesitamos aprender y concretar (mejor) la programación didáctica del departamento.

TABLA 3

¿Cómo se programa en el centro?

\begin{tabular}{|c|c|}
\hline PROFESORADO & EQUIPO DIRECTIVO \\
\hline $\begin{array}{l}\text { - Hacemos a nivel de departamento la pro- } \\
\text { gramación didáctica y sobre ella avan- } \\
\text { zamos utilizando como apoyo básico el } \\
\text { libro de texto. } \\
\text { - Desarrollamos algunas unidades didácti- } \\
\text { cas, pero nos falta concretar y aprender } \\
\text { sobre esta temática. } \\
\text { - A nivel programador nos apoyamos } \\
\text { mucho en los maestros del centro. }\end{array}$ & $\begin{array}{l}\text { - Sería necesario hacer un trabajo más } \\
\text { metódico a nivel programador, desde lo } \\
\text { que se establece en el diseño por parte } \\
\text { de la Administración educativa hasta su } \\
\text { mayor concreción curricular. } \\
\text { - En general, unos programan más, otros } \\
\text { menos... }\end{array}$ \\
\hline
\end{tabular}




\section{Grupo de discusión}

Los participantes en el grupo: equipo directivo, orientador escolar, profesorado de los departamentos de lengua castellana y literatura y matemáticas, han manifestado:

- Se programa y se enseña bien, aunque necesitamos formación y asesoramiento en este campo.

- Los maestros nos ayudan en esta tarea, pues su preparación pedagógica es mayor, aunque, en general, como profesores de enseñanza secundaria queremos aprender y avanzar en este tema.

TABLA 4

¿Se programa en distintos niveles de contextualización curricular?

\begin{tabular}{|c|c|}
\hline PROFESORADO & EOUIPO DIRECTIVO \\
\hline $\begin{array}{l}\text { - No, casi todos actuamos de la misma manera } \\
\text { en este campo de concreción curricular. } \\
\text { - Nos basamos, sobre todo, en el libro de texto. } \\
\text { - Necesitamos modelos de programación y } \\
\text { tiempo. } \\
\text { - Sería necesario programar a nivel de grupo y } \\
\text { con mayor precisión la programación didáctica } \\
\text { general, con vistas a su adaptación y contex- } \\
\text { tualización progresivas. }\end{array}$ & $\begin{array}{l}\text { - Unos profesores sí y otro no. En } \\
\text { general, hay que trabajar bastante } \\
\text { este tema. } \\
\text { - Desde la jefatura de estudios se } \\
\text { insiste en la necesidad de cambiar } \\
\text { las prácticas programadoras a tra- } \\
\text { vés de uu. dd. base y su secuencia- } \\
\text { ción posterior al alumnado. }\end{array}$ \\
\hline
\end{tabular}

\section{Grupo de discusión}

Los participantes en el grupo: equipo directivo, orientador escolar, profesorado de los departamentos de lengua castellana y literatura y matemáticas, han manifestado:

- Se programa lo justo y casi todo se reduce a la programación didáctica de cada departamento, aunque hay profesorado que avanza muy bien en este asunto.

- Hay que fomentar una nueva cultura de avanzar en el proceso de contextualización curricular para reducir el fracaso escolar (orientador).

- Sería conveniente realizar formación y autoformación en este asunto. 
LA UNIDAD DIDÁCTICA (INTEGRADA): DOCUMENTO PROGRAMADOR PARA

LA INNOVACIÓN-INDAGACIÓN EDUCATIVA(S) EN/DESDE UNA PERSPECTIVA INCLUSIVA. UN ESTUDIO DE CASO

TABLA 5

¿Se trabaja a nivel de programación por unidades didácticas?

\begin{tabular}{|c|c|}
\hline PROFESORADO & EQUIPO DIRECTIVO \\
\hline $\begin{array}{l}\text { - No en todos los casos. Hay profesorado que sí lo } \\
\text { hace, aunque se siguen las (unidades didácticas) } \\
\text { establecidas en el libro de texto. } \\
\text { - Hay una gran inquietud en aprender a progra- } \\
\text { mar mediante el diseño de unidades didácticas, } \\
\text { en general. } \\
\text { - Se demanda, sobre todo, por el profesorado de } \\
\text { lengua castellana y literatura y matemáticas un } \\
\text { modelo-base y operativo para contextualizar la } \\
\text { programación, y a ser posible mediante el diseño } \\
\text { de unidades didácticas. } \\
\text { - Hay que ser formados en este asunto, bajo la coor- } \\
\text { dinación del equipo directivo. }\end{array}$ & $\begin{array}{l}\text { - El profesorado, al igual que } \\
\text { nosotros en nuestra labor } \\
\text { docente, hacemos lo que sabe- } \\
\text { mos y podemos, pero necesi- } \\
\text { tamos asesoramiento por parte } \\
\text { de la Inspección educativa y } \\
\text { formación específica para el } \\
\text { trabajo por unidades didácticas. } \\
\text { - Hay profesorado que sí trabaja } \\
\text { (muy asiduamente) por unida- } \\
\text { des didácticas, aunque no sabe } \\
\text { si está bien o mal. }\end{array}$ \\
\hline
\end{tabular}

\section{Grupo de discusión}

Los participantes en el grupo: equipo directivo, orientador escolar, profesorado de los departamentos de lengua castellana y literatura y matemáticas, han manifestado:

- Hacemos lo que podemos y nos ponemos de acuerdo a nivel de departamento, aunque es preciso mejorar en esta línea de trabajo (unidades didácticas), además saber unir las mismas con el currículo previamente establecido por la Administración educativa.

- Este aspecto es fundamental en los dos primeros cursos de la ESO, pues, en cierta forma, se evitaría un gran porcentaje de fracaso escolar.

- Sería muy conveniente para las áreas instrumentales de lengua castellana y literatura y matemáticas.

TABLA 6

¿Se trabaja a nivel interdisciplinar y por unidades didácticas en el 1.er ciclo de la ESO?

\begin{tabular}{|c|c|}
\hline PROFESORADO & EQUIPO DIRECTIVO \\
\hline $\begin{array}{l}\text { - Lo que se refiere a las unidades didácticas, ya se ha } \\
\text { dicho antes; en lo referente a la interdisciplinariedad } \\
\text { no se trabaja prácticamente y no tenemos tiempo para } \\
\text { dicha modalidad curricular. } \\
\text { - La interdisciplinariedad exigen un gran trabajo en } \\
\text { equipo y necesitamos más tiempo para ello; aunque } \\
\text { sería muy productiva para determinados temas. } \\
\text { - Necesitamos formación y asesoramiento para determi- } \\
\text { nar cuándo procede dicha forma de trabajar a nivel } \\
\text { curricular. }\end{array}$ & $\begin{array}{l}\text { - Muy poco y determina- } \\
\text { dos momentos, hay que } \\
\text { preparar al profesorado } \\
\text { en esta opción didáctica. } \\
\text { - Sería conveniente adqui- } \\
\text { rir formación en esta } \\
\text { faceta a través de los cen- } \\
\text { tros de profesorado. }\end{array}$ \\
\hline
\end{tabular}


Grupo de discusión

Los participantes en el grupo: equipo directivo, orientador escolar, profesorado de los departamentos de lengua castellana y literatura y matemáticas, han manifestado:

- En general, no se trabaja dicha opción metodológica, aunque hay un 20 \% del profesorado que sí lo hace de forma esporádica en los dos primeros cursos de la Eso.

- Hay que formarse en esta faceta para potenciar ciertas competencias escolares (comunicativo-lingüística, sobre todo).

- Necesitamos formación, asesoramiento y tiempo.

TABLA 7

¿Se trabaja la lectura de forma integrada a nivel curricular?

\begin{tabular}{|c|c|}
\hline \multicolumn{1}{|c|}{ PROFESORADO } & \multicolumn{1}{c|}{ EQUIPO DIRECTIVO } \\
\hline - En general, no. Se dedica un tiempo \\
específico como determina la norma, \\
$\begin{array}{l}\text { aunque de forma específica en el ámbito } \\
\text { de cada materia. }\end{array}$ & $\begin{array}{c}\text { - Sería muy necesario, pues ayudaría a } \\
\text { reducir el fracaso escolar, entre otros } \\
\text { aspectos. }\end{array}$ \\
- Sería conveniente diseñarla de forma \\
$\begin{array}{l}\text { Necesitamos formación y una posterior } \\
\text { integrada: se fomentaría la habilidad } \\
\text { comprensiva y otras asociadas a ella. }\end{array}$ & $\begin{array}{l}\text { lengua castellana y literatura, sobre todo } \\
\text { para trabajar en el primer ciclo de la ESO. }\end{array}$ \\
\hline
\end{tabular}

\section{Grupo de discusión}

Los participantes en el grupo: equipo directivo, orientador escolar, profesorado de los departamentos de lengua castellana y literatura, matemáticas y delegados de grupo de los dos primeros niveles de la ESO (alumnado), han manifestado:

- El 90 \% del profesorado lo ve como una opción muy positiva, aunque no saben cómo hacerlo.

- El departamento de lengua castellana y literatura está muy interesado en esta cuestión, pues se fomentaría la lectura comprensiva y el hábito lector de una forma muy diferente a como normalmente te hace.

- El alumnado, en un $70 \%$, manifiesta su interés en esta faceta lectora, pero partiendo, sobre todo, de bloques que sean de su interés.

Como resultado final se ha expuesto un modelo de referencia programadora en/para el IEs -estudio de caso-, para seguir trabajando con él, a lo largo de los siguientes/próximos cursos escolares, en un intento de reformarlo y regenerarlo por parte del claustro, ante la validez dada al mismo por el equipo técnico de coordinación pedagógica/comision de coordinación pedagógica. Dicho modelo se adjunta en el anexo de este trabajo.

También, una vez establecidas las premisas de fundamentación metodológica (a nivel general), se elaboró el siguiente diseño de uD (docente-discente), desde la perspectiva metodológica integrada, infra. 
LA UNIDAD DIDÁCTICA (INTEGRADA): DOCUMENTO PROGRAMADOR PARA

LA INNOVACIÓN-INDAGACIÓN EDUCATIVA(S) EN/DESDE UNA PERSPECTIVA INCLUSIVA. UN ESTUDIO DE CASO

\subsection{La interdisciplinariedad en el proceso programador-contextual de los grupos- aula. Desarrollo}

De forma genérica, puede definirse la interdisciplinariedad como la agrupación de los contenidos de la enseñanza por temas, más que por separación por áreas/materias o disciplinas específicas (Salvador, Rodríguez y Bolívar, 2004).

Constituye esta segunda experiencia una "segunda» investigación (objetivo núm. 2) más focalizada siendo (desde la perspectiva del aprendizaje del alumnado) igualmente extensible al resto de niveles educativos y docentes del centro, en comparación con la primera, sobre diseños y desarrollos base de programación didáctica en una progresiva contextualización al grupo-aula.

En relación a este trabajo más focal y, partiendo de los elementos de referencia teóricos, ya estructurados anteriormente, se pretendió, por una parte, fomentar un trabajo colaborativo entre un número más reducido de docentes que en la primera investigación (objetivo núm. 1), supra, y, por otra parte, redundar en la propia mejora del aprendizaje de una muestra de alumnado, apático hacia el aprendizaje escolar, así como con necesidad específica de apoyo educativo (en general), siempre desde la mejora de (una actuación metodológica) y (de) la inclusión escolar (atención a la diversidad).

La finalidad fundamental de esta nueva opción investigadora ha girado en torno a los siguientes vectores de dirección, desde una perspectiva pedagógico-didáctica:

- Establecer una acotación del trabajo didáctico a cinco grupos escolares, dos de primero, dos de segundo y uno de tercero de EsO, respectivamente, en relación a un núcleo temático -unidad didáctica de referencia ${ }^{3}$, desde la perspectiva de la interdisciplinariedad-objetivo de investigación núm. 2, supra-;

- Generar un fraccionamiento de dicho núcleo temático a lo largo de un curso escolar, en aras de desarrollar un automatismo y acomodación del alumnado en/a los objetivos propuestos;

- Diseñar los bloques de referencia instrumentales, en las áreas/materias de lengua castellana y matemáticas, y sus programas de refuerzo en los niveles $1 .^{\circ}$ y $2 .^{\circ}$, en relación a la tipología del alumnado con el que se va a trabajar, tomando como referencia las competencias clave (lingúistica y matemática), como derivación de las pruebas previamente aplicadas, bien de forma estandarizada, bien por el profesorado (a modo de evaluación inicial y procesual);

- Centrar el trabajo como un estudio de caso, con referencia al centro docente y, por ende, al profesorado que indaga e innova en este campo de la didáctica;

3. UD: La lectura como eje programador en el centro. Su interdisciplinariedad. Ejemplificación en primer ciclo de la ESO. Acomodación a los distintos niveles por los respectivos equipos docentes. 
- Posibilitar la apertura funcional de esta investigación al resto de docentes interesados, en función de los resultados y de las condiciones pedagógicas de los grupos de discentes para cursos posteriores; e

- Implicar de forma sistemática al orientador del centro educativo.

A un nivel más descriptivo y gráfico, ver Figura 2, infra:

FIGURA 2

Metodología didáctica: desarrollo

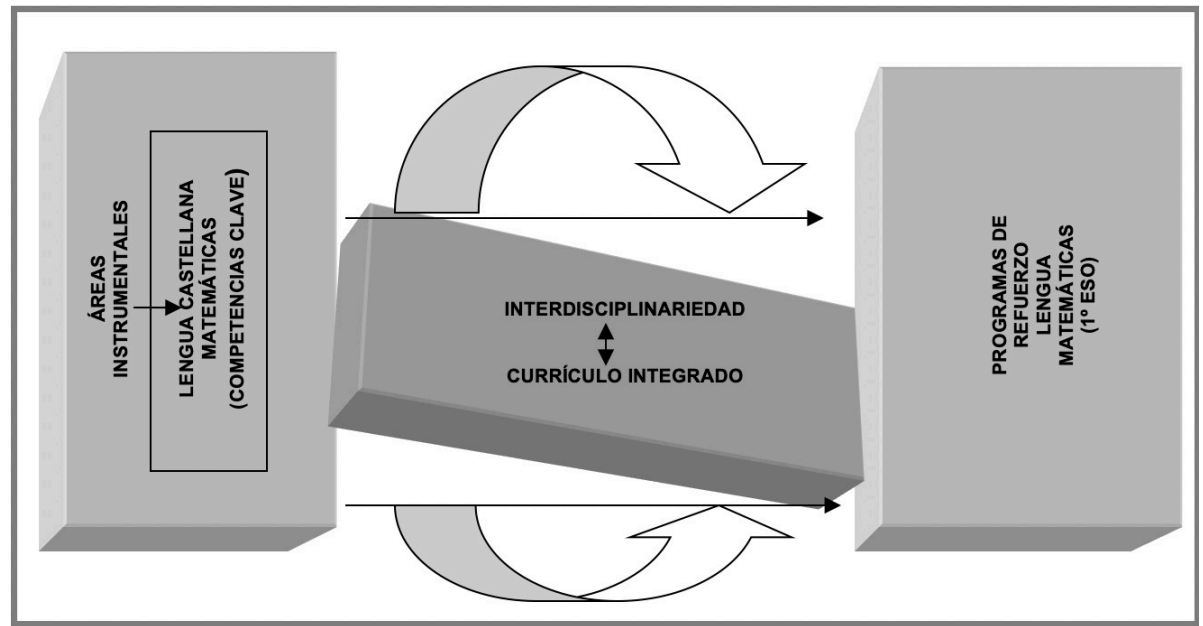

Como docentes implicados en esta nueva opción investigadora (objetivo núm. 2) se han determinado, entre otros, los siguientes:

- Una profesora de lengua castellana y literatura del Cuerpo de Maestros (597), adscrita a los dos primeros niveles de la ESO;

- Un profesor de lengua castellana y literatura del Cuerpo de Profesores de Enseñanza Secundaria (590);

- Un profesor de matemáticas del Cuerpo de Maestros (590), adscrito a los dos primeros niveles de la ESO;

- Un profesor de matemáticas del Cuerpo de Profesores de Enseñanza Secundaria (590);

- Las jefaturas de departamento de las respectivas materias/áreas;

- Un profesor de enseñanza secundaria en el programa de refuerzo de matemáticas (1. ${ }^{\circ}$ de la ESO);

- Un maestro en el área/materia de refuerzo de lengua castellana y literatura (1. ${ }^{\circ}$ de la ESO); 
LA UNIDAD DIDÁCTICA (INTEGRADA): DOCUMENTO PROGRAMADOR PARA

LA INNOVACIÓN-INDAGACIÓN EDUCATIVA(S) EN/DESDE UNA PERSPECTIVA INCLUSIVA. UN ESTUDIO DE CASO

- Un profesor de geografía e historia del Cuerpo de Maestros (590), adscrito a los dos primeros niveles de la ESO;

- La profesora de apoyo a la integración, Cuerpo de Maestros (597), para el alumnado con dificultades de aprendizaje en los dos primeros niveles de la ESO; y

- El orientador escolar.

Como núcleo temático general se ha diseñado, después de un muestreo (aleatorio) en el alumnado del primer ciclo de la educación secundaria obligatoria, el denominado: La no violencia y la paz, que, por otra parte, presenta una finalidad transversal en el currículo globalmente considerado, aunque la finalidad es presentarlo de forma imbricada en el desarrollo curricular de las áreas/materiascompetencias clave ya mencionadas.

Las fases de este proceso investigativo, así como las técnicas utilizadas, son coincidentes con las de la primera fase de esta amplia investigación (objetivo núm. 1), aunque se ha implementado el enfoque individualizador-personalizador de la enseñanza, así como sus correspondientes métodos de enseñanza en una visión (más) artística del desarrollo de los procesos de enseñanza-aprendizaje.

La finalidad del proceso didáctico ha sido establecer una integración, desde una visión psicológica, así como, desde la perspectiva pedagógica, estableciendo relaciones entre las diferentes materias/áreas, con la especificad de las mismas, en relación a un bloque temático común.

La programación base resultante en este fase investigadora (objetivo núm. 2), infra, ha sido la siguiente, sin menoscabar su transferencia ecológica a través de una investigación más profunda, haciendo, por ende, extensible todo el proceso al resto de posibles docentes, estableceiendo al mismo tiempo contrastes cualitativos entre distintos centros educativos.

\subsubsection{Programa: Desarrollo de la uD (integrada)}

Título: La lectura como eje programador en el centro. Su interdisciplinariedad y contextualización progresiva(s).

Bloque/Núcleo temático: La no violencia y la paz. Desarrollo cuatrimestral, febrerojunio (del año escolar afectado).

Su objeto, sus objetivos, población, meta, duración del programa, etc.:

Todo programa educativo (UD) debe entenderse como un plan sistemático de intervención, específica e intencionalmente elaborado, al servicio de metas consideradas valiosas desde una perspectiva pedagógico-didáctica (Martínez, 2017). En este sentido, y desde la nueva relevancia dada a la lectura en la LOE-TC y en el reglamento que la desarrolla en ESO (a nivel de currículo básico) ${ }^{4}$, la lectura se

4. Ver, en este sentido, Real Decreto 1105/2014, de 26 de diciembre, ya citado supra. 
nuclea como una habilidad básica para la reordenación funcional de las restantes materias en la ESO.

Como objeto (finalidad principal) se ha establecido la siguiente intención: Imbricar la lectura, como habilidad básica, del aprendizaje del alumnado en todas las áreas/materias, sensibilizando en esta tarea a todos los equipos docentes. Se utiliza como núcleo temático de referencia el reseñado anteriormente.

Los objetivos derivados de esta actuación general se han estratificado de la siguiente manera:

- Fomentar una implicación activa de los docentes en el ejercicio lector de los aprendices.

- Centralizar esta actuación desde la lengua castellana y Literatura, al resto de las áreas/materias de la ESO.

- Potenciar el trabajo escolar (en toda su amplitud) desde la lectura: lectura comprensiva.

- Propiciar, en última instancia, un hábito lector en el alumnado, mediante la creación de una biblioteca (de aula) -leer para aprender-.

El proceso de desarrollo de esta experiencia (centrada en la población de ESO) se ha establecido en el IES (estudio de caso), en los niveles $1 .^{\circ}$ a $3 .^{\circ}$, con una población de 200 educandos.

La finalidad curricular y metodológica ya establecida ha sido mejorar la lectura como competencia básica en el aprendizaje académico.

La duración de este programa corresponde al semestre enero/junio del año del proceso investigador.

El proceso metodológico-investigador ha obedecido a las siguientes fases:

1. Análisis de la puesta en marcha (justificación y problema)

En primer lugar, se ha tratado de sensibilizar al claustro sobre esta experiencia (en cierto modo ya imperativa por la LOE-TC), a través del equipo técnico de coordinación pedagógica, así como por el profesorado de los departamentos de lengua castellana y literatura, geografía e historia y matemáticas. En consecuencia, dicho profesorado ante las deficiencias detectadas en la lectura en sus distintas áreas/materias ha demandado dicha actuación. Establecida esta premisa se ha actuado y se actúa de la siguiente manera: 1) elaboración una temporalización mensual para el seguimiento de los aspectos básicos de la lectura comprensiva, diseñados por el departamento de lengua castellana y literatura y el departamento de orientación del IES; 2) preparación de un material específico para el desarrollo de la lectura por el departamento de lengua y literatura (preferentemente en el primer ciclo de la ESO, niveles $1 .^{\circ} \mathrm{y}$ 2. ${ }^{\circ}$ ), para su desarrollo en las distintas áreas/materias, tutorías como técnica de trabajo intelectual y para el alumnado disruptivo en el aula de convivencia; 3) la responsabilidad se deriva en los siguientes agentes: en general, todos los docentes del centro y familias a través de la orientación familiar (cuando 
proceda), en particular, los miembros del departamento de Lengua Castellana y Literatura y el orientador del centro y por extensión todo el departamento de orientación, bajo la supervisión de la jefatura de estudios, y 4) aportación de información permanente a toda la institución educativa sobre el desarrollo del programa, así como solicitando el asesoramiento correspondiente de la Inspección educativa y el Centro de Profesorado de la zona.

2. Observación participante (descripción y conclusiones fundamentadas). Cuaderno de campo. Trabajo docente-discente a nivel de colegas

Una vez diseñados los objetivos y contenidos de una forma interdisciplinarintegrada, se ha procedido por parte del orientador y la jefatura del departamento de lengua castellana y literatura a la interacción y aportación con los docentes de referencia, a nivel de colegas, posibilitando textos (sobre la no violencia y la paz) para ejercitar la lectura comprensiva, así como el ejercicio del hábito lector en los aprendices tanto en dicha materia cuanto en otras de su propio interés.

En esta tarea se han establecido distintos niveles funcionales de actuación:

- Trabajo reflexivo-autónomo sobre el proceso lector, con lecturas propuestas por los propios aprendices (método colaborativo);

- Se ha procedido a la lectura (magistral-modelo) por parte del docente y de determinados educandos;

- Se ha personalizado el aprendizaje en cada estudiante de acuerdo a su singularidad individual; y

- Se han establecido grupos de investigación-innovación para el alumnado y docentes-discentes, con la finalidad de generar nuevas lecturas y modalidades de indagación en otros parámetros (de perfeccionamiento) del proceso lector: exactitud, entonación y velocidad.

En última instancia se han establecido torneos entre grupos, extrapolando los textos a otras áreas, con la finalidad de ejercitar otro tipo de competencias: algoritmos, resolución de problemas, etc.

En nuestro trabajo y a nivel de colegas hemos elaborado un cuaderno de campo [ver Cuadro 1, infra], en aras de describir todos los aspectos de mejora y reforma del proceso lector. En este ámbito hemos utilizado distintas técnicas evaluadoras a nivel de aprendizaje y enseñanza: coevaluación, heteroevaluación y autoevaluación. Por otra parte, se procedió al análisis del aprendizaje, a la vista de los intrumentos y estrategias diseñados en la página 5 (Anexo de este trabajo).

3. ¿Con qué finalidad realizaría la evaluación de la UD (o función de la evaluación)?

El proceso evaluador debe propiciarnos una información cualitativa de la siguiente naturaleza: 1) detectar deficiencias en su desarrollo y retroalimentarlas de forma inmediata-mediata; 2) obtener información sobre el desarrollo 
CUADRO 1

Cuaderno de campo

\begin{tabular}{l}
$\begin{array}{l}\text { Sesión de trabajo, } \mathrm{n}^{0}: \_ \text {I Fecha: } \\
\text { Agentes docentes: } \\
\text { Actividades: descripción: }\end{array}$ \\
- Incidencias en el proceso lector: \\
- Parámetros afectados: \\
- Desarrollo UD: \\
- Evaluación del proceso: \\
- Conclusiones: \\
- Reuniones de trabajo posteriores: \\
\hline
\end{tabular}

Elaboración personal.

de lo planificado (de forma global); 3) determinar el grado de implicación y cumplimiento de todos los agentes (responsabilidad del jefe de estudios); 4) analizar el grado de satisfacción en el alumnado, en relación al desarrollo del programa y cumplimiento de sus expectativas; 5) de forma análoga, para los docentes implicados, 6) valorar la información obtenida en la continuidad evaluadora del programa como base para su mejora y toma de decisiones.

4. ¿Qué tipo de información recogería para conocer cómo funciona el programa? (En relación con la vida del programa).

La información debe ser obtenida con vistas a una interpretación cualitativa de la misma en relación a las siguientes características: a) Información inicial: se deben analizar aspectos tales como seriedad de la planificación, desarrollo en la primera quincena de aplicación, corresponsabilidad inicial de todos los agentes educativos...; b) Información de proceso: debe establecerse por meses, con una valoración de los docentes-tutores de cada grupo-aula, así como por los jefes de los departamentos didácticos (afectos) y el orientador del centro, y c) Información de producto-resultado: debe establecerse de forma bimensual y al final para valorar la mayor o menor consecución de los objetivos didácticos planificados, proponiendo la continuidad del programa y su modificación (en su caso).

5. ¿Con quiénes contaría para recoger la información?

Dichos agentes ya han sido referenciados en las cuestiones anteriores; no obstante, es conveniente nombrar en cada grupo-aula un coordinador de lectura para evaluar el desarrollo del proceso a lo largo de todo el semestre. 
LA UNIDAD DIDÁCTICA (INTEGRADA): DOCUMENTO PROGRAMADOR PARA

LA INNOVACIÓN-INDAGACIÓN EDUCATIVA(S) EN/DESDE UNA PERSPECTIVA INCLUSIVA. UN ESTUDIO DE CASO

\section{6. ¿En qué momentos recogería la información?}

De forma análoga, esta cuestión ya ha sido respondida. Reiteramos que la información debe ser recogida de forma continua y de una forma más sistemática (para una valoración formativa del programa) de forma bimensual y al final de su aplicación (mes de junio del correspondiente curso escolar).

7. ¿Qué técnicas de recogida de información e instrumentos utilizaria?

La información del desarrollo del programa debe ser recogida e informada posteriormente para su mejora. Es necesario tener en cuenta, a priori, que las técnicas y los instrumentos son términos-conceptos interrelacionados, aunque en el desarrollo de los procesos educativos sean «técnicamente» diferentes. Por los límites de la extensión de este trabajo no vamos a profundizar en ambos términos, aunque sí diferenciaremos la exposición (para la recogida de la información), en relación a ambos términos [ver Cuadro 2, infra].

CUADRO 2

Técnicas e instrumentos de investigación educativa

\begin{tabular}{|c|c|}
\hline TÉCNICAS EDUCaTIVAS & INSTRUMENTOS EDUCATIVOS \\
\hline $\begin{array}{l}\text { 1. Recogida directa de información. } \\
\text { 2. Recogida de información. } \\
\text { 3. Comprobaciones y contrastes. } \\
\text { 4. Análisis documental. } \\
\text { 5. Transferencia del núcleo trabajado a } \\
\text { otros contextos con el uso de las Tic, } \\
\text { por parte del profesorado y alumnado. }\end{array}$ & $\begin{array}{l}\text { 1'. Guiones de observación participante. } \\
\text { 2'. Escalas y fichas. } \\
\text { 3'. Cuestionarios y escalas de calificación. } \\
\text { 4'. Cuadernos de clase y trabajoprocedimental. }\end{array}$ \\
\hline
\end{tabular}

Elaboración personal.

La elaboración de todo este trabajo debe ser, en todo momento, cooperativa-reflexiva.

8. ¿Qué criterios de evaluación utilizaría?

Obviamente, debemos centrar nuestro proceso evaluador en la globalidad del programa educativo, procediendo progresivamente a un descenso del trabajo operativo al grupo-aula. Es evidente que todas las etapas de aplicación del programa están interrelacionadas. Los criterios deben establecerse desde el equipo técnico de coordinación pedagógica para transferirse posteriormente a todos los órganos de coordinación docente. En todo momento, dichos criterios están abiertos a su modificación, de acuerdo al desarrollo continuo del programa implementado.

Como criterios establecemos los siguientes:

- Coherencia y fiabilidad del programa diseñado (para la mejora de la lectura en la ESO);

- Simplicidad y concreción-operativización del mismo; 
- Secuenciación de la competencia comunicativo-lingüística a nivel de la habilidad (lectura comprensiva);

- Interdisciplinariedad con otras disciplinas (áreas/materias) académicas;

- Transferencia de la mejora lectora a otros ámbitos «no escolares»;

- Implementación colaborativa-cooperativa por parte de todos los docentes;

- Motivación progresiva que genera en el propio alumnado, en relación a la compresión lectora; y

- Extensión de dicho aspecto lector a otros (complementarios) y paralelos en dicho proceso: velocidad, entonación y exactitud lectora.

9. ¿Cómo aplicaría los criterios para evaluar el programa?

La aplicación de los criterios anteriores exige (en el desarrollo del proceso del programa educativo) establecer de forma colegiada un análisis de "tareas" de los mismos. No obstante, de forma sintetizada podemos establecer la siguiente metodología de aplicación-valoración de los criterios ya referenciados.

En primer lugar, debe procederse a correlacionar la habilidad básica (lectura) con los objetivos-criterios de evaluación establecidos en las programaciones didácticas de los diferentes departamentos didácticos, con preferencia en el correspondiente a lengua castellana y literatura. De forma análoga, debe transferirse dicho proceso a la progresiva contextualización curricular de todos los docentes. Esta información se debe recoger en los resultados de la evaluación continua del alumnado. En otra fase y en paralelo la jefatura de estudios junto con el departamento de orientación elaboraría escalas de seguimiento y cumplimiento de los parámetros generales del programa educativo [coherencia, implicación colectiva docente, realidad y concreción...], detectando deficiencias y estableciendo las consiguientes mejoras. Por último, en un claustro, debe establecerse una autoevaluación de los criterios diseñados para su rectificación y adaptación del proceso.

10. ¿Cómo comunicaría los resultados de la evaluación y a quiénes la daría? Los resultados deben tener una visión formativo-orientadora para la continuidad del proceso programador en este ámbito conceptual.

La publicidad de los resultados debe establecerse en los siguientes niveles de actuación:

- Realización de un informe técnico por parte del equipo técnico de coordinación pedagógica, para conocimiento de todos los departamentos;

- Estudio de dicho informe por parte del equipo directivo y reelaboración de un informe global para su extrapolación al claustro (para la posterior toma de decisiones);

- Envío de dicho informe de la dirección a la Inspección educativa, para un asesoramiento posterior (si fuese necesario);

- Traslación de la experiencia a la comunidad educativa (a través de su órgano de máxima representación: Consejo Escolar); y

- Reflexión, a pie de aula, con el alumnado por parte de todos los docentes, con especial relevancia en los tutores y profesorado de lengua castellana y 
LA UNIDAD DIDÁCTICA (INTEGRADA): DOCUMENTO PROGRAMADOR PARA

LA INNOVACIÓN-INDAGACIÓN EDUCATIVA(S) EN/DESDE UNA PERSPECTIVA INCLUSIVA. UN ESTUDIO DE CASO

literatura, reflejando los resultados con los cuadernos de trabajo, pruebas, etc.

\section{CONCLUSIONES (FINALES) Y DISCUSIÓN}

Como conclusiones finales, en todo el proceso de investigación en sus dos niveles (objetivos núms. 1 y 2) podemos establecer, entre otras, las siguientes: Adaptación de la UD.

- Se ha mejorado el trabajo colaborativo y la implicación docente en su faceta programadora a nivel progresivo y contextual;

- Se ha estimulado y activado indirectamente a otros docentes de los departamentos afectados, principalmente, en tercero de la ESO;

- El alumnado ha mejorado su nivel de competencia curricular y, por ende, en la adquisición de sus rendimientos escolares, en los aspectos básicos referidos a las áreas instrumentales;

- Se ha generado una visión de la didáctica más constructiva, novedosa, enriquecedora y armoniosa para el trabajo docente; todo ello, sobre todo, desde una visión metodológica y evaluadora;

- Se ha conseguido integrar y "unir» áreas/materias afines, aunque de diferente naturaleza y organización conceptual;

- Se ha generado una nueva visión de la propia orientación educativa, a nivel metodológico;

- Se ha estimulado al profesorado a generar proyectos de innovación educativa en este campo, a tenor de las convocatorias oficiales de la Administración educativa competente; $y$

- Se ha generado una transferencia del trabajo metodológico desarrollado a los centros de la zona mediante una actuación específica de la Inspección educativa.

- Mediante el seguimiento de la experiencia realizada (un semestre) se ha reducido el número de suspensos en las áreas instrumentales, habiendo un incremento de un $40 \%$ en el número de educandos que han abandonado los programas de refuerzo en $1 .^{\circ}$ de la ESO, en lengua castellana y literatura.

- Finalmente, se han activado las bibliotecas de aula en determinados grupos escolares, como parte del plan lector del centro, todo lo cual ha redundado en un aumento significativo de la habilidad lectora a nivel comprensivo.

Se demanda por parte de todo el profesorado intensificar esta experiencia a lo largo de los próximos cursos, con la participación de expertos en programación curricular, a través de las distintas modalidades de formación docente, con la finalidad de estabilizar esta buena práctica programadora. Además, se ha procedido a colegir el modelo-base de programación contextualizada con aquellos elementos 
curriculares que son esenciales en el mismo, estableciendo el procedimiento de cumplimentación más operativo al efecto; no obstante, se concluye que dicho modelo será dinámico y que se irá regenerando conforme se vaya aplicando, en aquellos aspectos que se considere necesario.

\section{ANEXO}

\section{EL PROCESO DE CONTEXTUALIZACIÓN CURRICULAR (GRUPOS-AULA)}

El proceso programador en el ámbito escolar exige establecer distintos niveles de concreción y contextualización curricular, siendo el último de estos -contextualización en los grupos-aula- el que reorganiza, define, concreta e interpreta la acción educativa en cada grupo-aula, atendiendo a las características específicas del alumnado.

Los docentes, dentro de sus respectivos equipos educativos, departamentos didácticos..., deben programar su actividad docente, reordenando los distintos elementos curriculares, de forma autónoma y contextualizada, en un intento de adaptarlos a la realidad de cada grupo-aula y, por ende, de cada educando, de forma individualizada, personalizada, en el ambiente social que conforma el proceso de enseñanza-aprendizaje.

El profesorado debe programar su acción docente y su enseñanza como parte esencial de su praxis docente, en un ejercicio continuado de contextualización curricular; aspecto que ya se ha comentado supra, en la parte teórica de este trabajo, a nivel general, y, a nivel particular, para la ESO.

El presente documento responde a un formato de seis páginas-guía para programar la práctica educativa. Las seis páginas responden a los cuadros establecidos a normalizar en páginas A-4 (o en formato informatizado); de tal forma que el profesorado arbitrará la temporalización para cada programación establecida (quincenal mensual/quincenal para la ESO), así como la determinación de las unidades didácticas correspondientes.

Los objetivos se establecerán por siglas (letras y números), siendo los de aprendizaje específicos para cada grupo-aula e integradores de los generales y ciclo (si estos últimos han lugar); por ejemplo: Objetivo B (general) que trabaja primer curso de ciclo, .... . Los contenidos se establecerán de forma genérica por bloques dependiendo de cada unidad didáctica. Los aspectos metodológicos y de evaluación ya vienen definidos de forma genérica; deberán remarcarse algunas actividades-modelo-competenciales para cada unidad didáctica, así como remarcar en la página cinco con una $\mathrm{X}$ instrumentos, estrategias, etc., trabajados en la misma. Por último, en la página seis se le dará redacción a los objetivos didácticos que serán determinados y redactados para cada profesor, tanto en número como en contenido y con letras minúsculas, viendo la convergencia de toda la estructura curricular. 
LA UNIDAD DIDÁCTICA (INTEGRADA): DOCUMENTO PROGRAMADOR PARA

LA INNOVACIÓN-INDAGACIÓN EDUCATIVA(S) EN/DESDE UNA PERSPECTIVA INCLUSIVA. UN ESTUDIO DE CASO

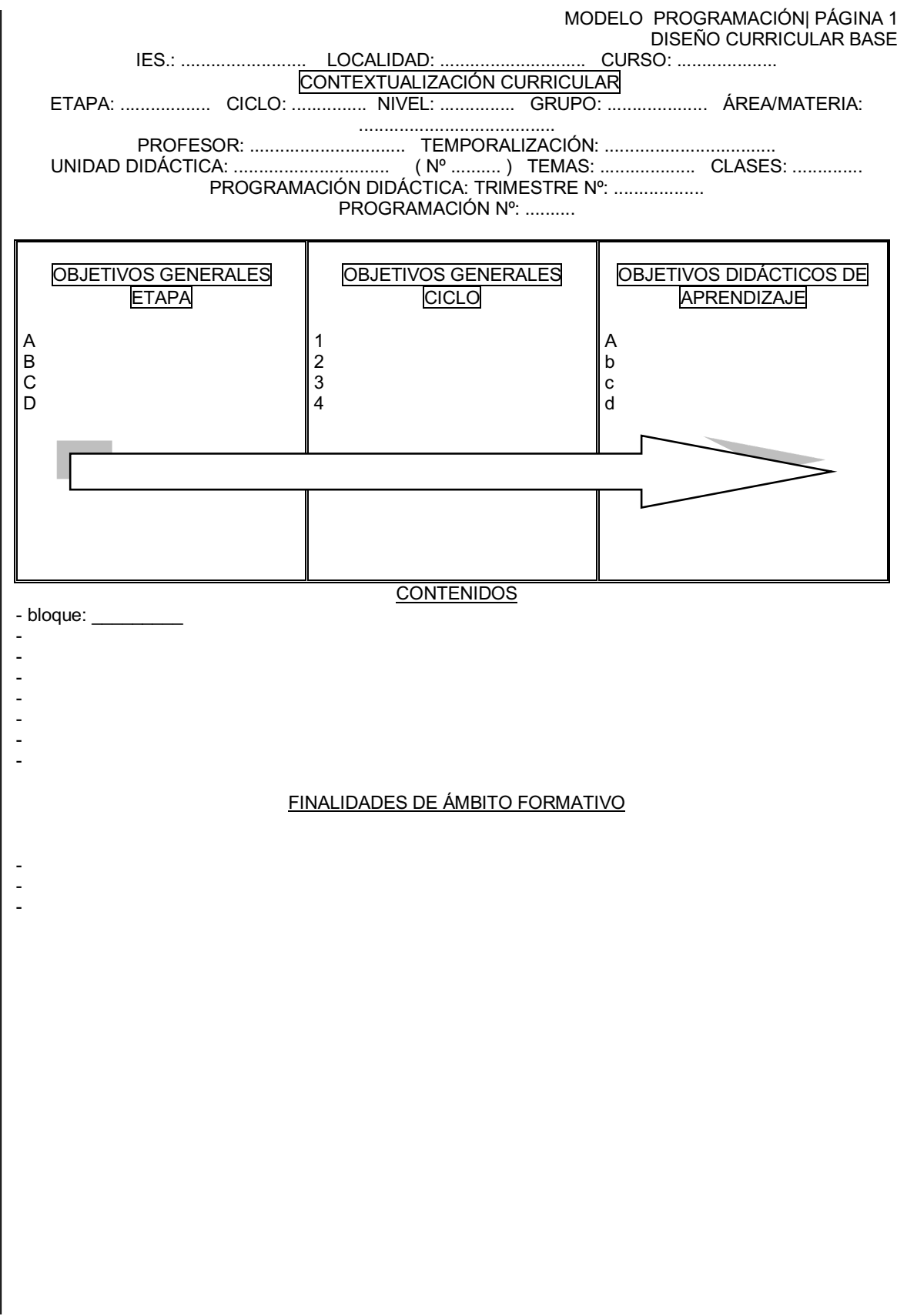




\section{METODOLOGÍA.}

Aspectos generales. La práctica diaria en los grupos-aula (a nivel de contextualización curricular) conlleva definir una serie de criterios metodológicos globales y específicos para cada área/ámbito que de forma progresiva contribuirán a facilitar el aprendizaje del alumnado, así como nuestra práctica docente.

La planificación global, por otra parte, facilitará el diseño de estrategias específicas para el desarrollo de cada área/ámbito, potenciando el trabajo autónomo del alumno, el aprender a aprender, etc.; se hace necesario, por ello, diseñar actividades-modelo-competenciales que faciliten la consecución de los diferentes contenidos, objetivos didácticos y competencias clave planteados para cada disciplina.

La diversidad existente en cada grupo-aula hará que tomemos medidas para atender posibles desfases en el aprendizaje del alumnado, bien mediante la modificación global o parcial del currículo, bien por la adopción de los correspondientes recursos materiales y tecnológicos disponibles en el centro.

Como grupo de trabajo se parte, en todo momento, de una coordinación global en vertical derivada de la programación didáctica realizada a nivel de departamento, así como también en horizontal a nivel de equipo educativo. En todo momento tenemos presente el currículo establecido para la ESO y bachillerato, así como su contextualización a las características del centro (proyecto educativo y programaciones didácticas a nivel de departamento); en consecuencia, se han definido los siguientes criterios generales para la organización del proceso enseñanza-aprendizaje en los diferentes grupos-aula:

- Partir del nivel de desarrollo del alumnado;

- Fomentar el trabajo autónomo, así como el aprendizaje significativo,

- Tomar en consideración el potencial de aprendizaje del alumnado, facilitando el aprender a pensar, así como el aprender a aprender;

- Favorecer el desarrollo integral del alumnado, fomentando el desarrollo de los temas transversales en el curriculum del área trabajada en cuestión.

- Posibilitar la reflexión sobre lo aprendido, haciendo hincapié en los aspectos procedimentales y actitudinales del currículo;

- Tomar en consideración los diferentes ritmos de aprendizaje del alumnado, readaptando en todo memento el curriculum desde una perspectiva metodológica;

- Favorecer distintos tipos de agrupamiento en el aula, dependiendo en todo momento de los contenidos a impartir;

- Favorecer la flexibilidad en los horarios y en los espacios a la hora de desarrollar el curriculum propuesto;

- Fomentar un trabajo interdisciplinar en determinadas áreas/ámbitos propuestas para/en la ESO;

- Favorecer todo tipo de recursos didácticos, procediendo los mismos del profesorado y del alumnado;

- Diseñar estrategias y procedimientos contextualizados a la realidad de cada grupo-aula, posibilitando la atención a la diversidad y la inclusión escolar;

- Tomar en consideración aquellas opciones metodológicas que sean propuestas por el alumnado, de forma individual o en grupo; $y$

- Temporalizar los contenidos a enseñar de forma flexible y constructiva.

La revisión de estas pautas globales en el diseño específico de cada área/ámbito a nivel metodológico debe contribuir a que el profesorado, de forma individual y grupal, readapte y modifique criterios globales y específicos como investigador de su propia práctica docente.

ASPECTOS ESPECÍFICOS - DISEÑO DE ACTIVIDADES-MODELO-COMPETENCIALES-

$$
\begin{array}{ll}
\hline \text { ASPECTOS ESPECÍFICOS - DISEÑO DE ACTIVIDADES-MODELO-COMPETENCIALES- } \\
- & - \\
- & - \\
- & - \\
- & - \\
- & -
\end{array}
$$




\section{EVALUACIÓN.}

Aspectos a evaluar. Definimos la evaluación como el elemento curricular por el cual establecemos juicios de valor sobre los procesos seguidos por el alumnado en su aprendizaje, así como los resultados obtenidos en dicho aprendizaje, tomando como referencia los objetivos generales de etapa/ciclo y los logros establecidos en los objetivos didácticos (secuenciados y contextualizados) a través de los criterios y estándares de evaluación previamente definidos. De forma análoga, la evaluación conllevará modificar el proceso de enseñanza y establecer valoraciones de carácter cualitativo y procesual de forma colegiada entre todo el profesorado. En definitiva, todo el proceso evaluador se llevará a cabo de forma continua y personalizada.

El aspecto procesual de la misma hará que el profesorado mediante determinadas estrategias y procedimientos analice de forma cualitativa y formativa los siguientes aspectos referentes al aprendizaje del alumnado:

- Implicación aptitudinal/actitudinal en los diferentes aprendizajes planteados;

- Estrategias que el propio alumnado utiliza para la resolución de los casos/problemas planteados por el profesorado:

- Participación, individual o en grupo, sobre los distintos contenidos a impartir, así como el planteamiento de otros no planificados;

- Autonomía en los diferentes aprendizajes, tomando como referencia el aprendizaje guiado establecido por el profesor;

- Evaluación de la propia enseñanza impartida por el profesor, en aras de una mejora cualitativa y práctica;

- Implicación de su formación integral y personal en el proceso diario de la clase (valoración de los temas transversales);

- Cooperación en el grupo para la consecución de los logros pretendidos;

- Consecución de las capacidades implicadas en las diferentes unidades implicadas, haciendo hincapié en el aprender a aprender.

De forma análoga, la verificación de todas las capacidades/competencias clave previstas para una determinada unidad didáctica, a través de los criterios de evaluación y actividades (previamente) establecidos, posibilitará que podamos hacer una medición [directa e indirecta] del proceso de aprendizaje del alumno (carácter sumativo de la evaluación), tomando como referencia base el aspecto formativo de la evaluación.

Momentos de la evaluación: La evaluación es un proceso continuo por su propia naturaleza formativa. No obstante, podemos diferenciar distintos "momentos" dentro de esta misma continuidad, que siempre serán referenciales para la globalidad del proceso evaluador. Así establecemos los siguientes:

- Evaluación inicial: determinación de pruebas de estado inicial, diferenciales por grupo-aula, para iniciar el proceso de aprendizaje del alumnado.

- Evaluación relativa a los diferentes logros de aprendizaje: se establece en función de los criterios (de evaluación) programados en cada unidad didáctica, tomando como referencia las capacidades/objetivos globales de etapa/ciclo, amén de los objetivos didácticos/unidad didáctica en el desarrollo de todas las áreas/materias de la ESO.

Cómo evaluar: La forma de evaluar organiza el aprendizaje de los alumnos, así como los propios métodos de enseñanza. Constituye el eje del proceso evaluador, debiendo programarse de una forma homogénea en la programación didáctica de departamento, ad hoc dar autonomía e individualidad al profesorado en sus diferentes procesos de contextualización curricular. Este aspecto debe abordarse bajo dos variables interrelacionadas a lo largo del proceso de enseñanza-aprendizaje, así:

Criterios de evaluación: Los criterios de evaluación son referentes generales para una mejor organización del proceso evaluador y del propio sistema de evaluación. Por otra parte, los criterios de evaluación serán prácticos y operativos, constando de un enunciado y una breve explicación en relación a los objetivos didácticos previamente establecidos.

En definitiva, establecen el tipo y grado de aprendizaje que se espera que alcance el alumnado en relación a las diferentes capacidades/objetivos establecidas en las diferentes áreas/materias y, por derivación pedagógica, en los distintos estándares de aprendizaje evaluables previstos para cada nivel/curso. 
En la elaboración de estos criterios se tendrán en cuenta los siguientes aspectos de proceso [colegiada e individualmente a nivel del profesorado]:

- Tendrán como referencia los criterios establecidos para las diferentes áreas/materias en los diferentes diseños curriculares base, tanto a nivel básico (Real Decreto 1105/2014, de 26 de diciembre, por el que se establece el currículo básico de la ESO y del bachillerato, como a nivel concreto-procedimental en el ámbito de los reglamentos establecidos al respecto por cada Comunidad Autónoma.

- Reflejarán los aprendizajes básicos de las áreas/materias, es decir aquellos que de no haber sido asimilados pueden impedir el progreso en el estudio de la áreas en una fase posterior del curso, o bien de cursos posteriores.

- No serán objetivos operativos, aunque en todo momento serán referenciales de los objetivos didácticos establecidos para la unidad didáctica, facilitando de forma permanente la orientación al alumnado en su proceso de aprendizaje. Por otra parte, tampoco deben coincidir en número con los objetivos didácticos planificados, siendo este un criterio que deba elaborar y decidir cada profesor bajo las directrices ya establecidas en del departamento didáctico, en coherencia con la programación (didáctica) previamente elaborada.

- Serán flexibles y permitirán obtener (sobre cada uno), una diversidad de actividades de evaluación que se adaptarán en todo momento a la diversidad del alumnado y recursos didácticos a emplear en cada grupoaula; siendo dichas actividades un referente evaluador a nivel indirecto de las competencias clave a conseguir a lo largo de la etapa (ESO).

- Se procederá al desarrollo de los mismos en diversas modalidades de evaluación, dependiendo de la situación aula: autoevaluación, heteroevaluación y coevaluación.

\section{CRITERIOS DE EVALUACIÓN REFERENCIALES}

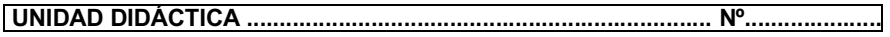




\section{MODELO PROGRAMACIÓN | PÁGINA 5 \\ PROCESO PROGRAMADOR BASE}

- Procedimientos y estrategias de evaluación: Planteamos para el diseño de la totalidad de unidades didácticas del presente curso escolar una matriz de referencia, estableciendo en la misma los diferentes aspectos implicados, así como los recursos de procedimiento y estrategias utilizados en cada momento con una (X); pretendemos de esta manera facilitar el trabajo, individual y colectivamente, así como facilitar la corrección del mismo sobre este documento base (ver, tabla de referencia, infra).

Tabla de referencia

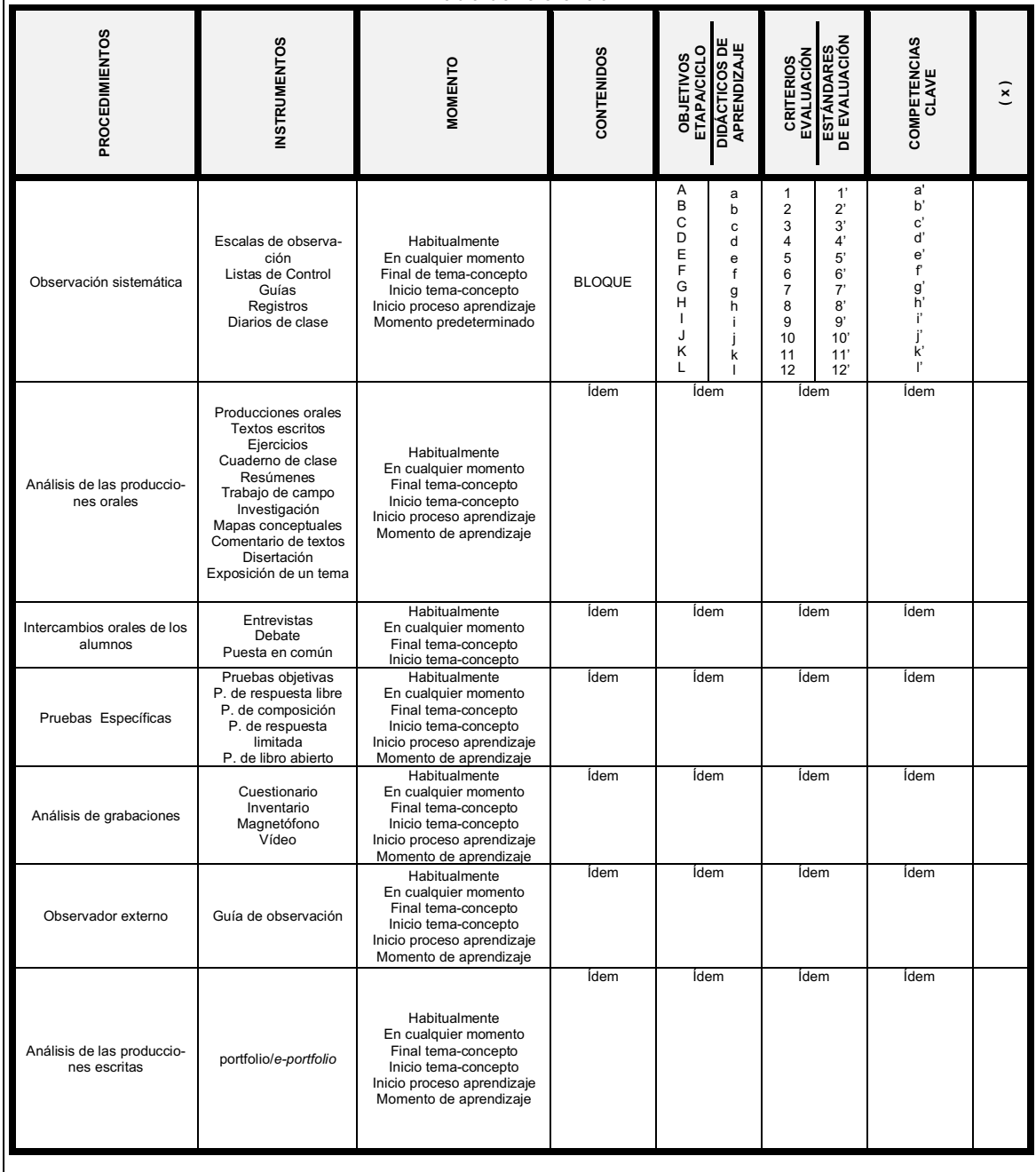


LA UNIDAD DIDÁCTICA (INTEGRADA): DOCUMENTO PROGRAMADOR PARA

LA INNOVACIÓN-INDAGACIÓN EDUCATIVA(S) EN/DESDE UNA PERSPECTIVA INCLUSIVA. UN ESTUDIO DE CASO

MODELO PROGRAMACIÓN | PÁGINA 6 PROCESO PROGRAMADOR BASE

\section{RELACIÓN DE OBJETIVOS DIDÁCTICOS CORRESPONDIENTES A LA}

U.D:

$\mathbf{N}^{\circ}$
a
b.
C.
d.
e.
f.
h.
i.
j.
k.
I.

g. 
LA UNIDAD DIDÁCTICA (INTEGRADA): DOCUMENTO PROGRAMADOR PARA

LA INNOVACIÓN-INDAGACIÓN EDUCATIVA(S) EN/DESDE UNA PERSPECTIVA INCLUSIVA. UN ESTUDIO DE CASO

\section{REFERENCIAS BIBLIOGRÁFICAS}

AA. VV. (1997). Indicadores educativos: Cinco opiniones. Cuadernos de Pedagogía (256), $70-75$

Aguaded, J. I. (2009). Nuevas formas de comunicación: cibermedios y medios móviles. Comunicar, 33, 10-13.

Arco, J. L., y Fernández, A. (Coords.). (2004). Necesidades Educativas Especiales. Manual de Evaluación e Intervención Psicológica. Madrid: McGraw-Hill.

Bronfenbrenner, (1985). Contextos de crianza del niño, problemas y perspectivas. Revista Infancia y Aprendizaje, 29, 51-52.

Cabrerizo, J. y Rubio, M. J. (2007). Atención a la diversidad. Teoría y práctica. Madrid: Pearson Educación.

Cardona, J. (2001). Elementos de Teoría Organizativa del Centro Escolar. Madrid: Sanz y Torres.

Casanova, M. A. (1992). La evaluación, garantía de calidad para el centro educativo. Zaragoza: Edelvives.

Escamilla, A. (1993). Unidades didácticas: una propuesta de trabajo en el aula. Zaragoza: Edelvives.

España. Ley Orgánica 2/2006, de 3 de mayo, de Educación, Texto Consolidado. Boletín Oficial del Estado, Legislación Consolidada, núm. 295, 10 de diciembre de 2013.

España. Ley Orgánica 2/2006, de 3 de mayo, modificada por la Ley Orgánica 8/2013, de 9 de diciembre [Loe-Texto Consolidado]. Boletín Oficial del Estado, 4 de mayo de 2006, núm. 106. Referencia: BOE-A-2006-7899.

España. Ley Orgánica 8/2013, de 9 de diciembre, para la mejora de la calidad educativa. Boletín Oficial del Estado, núm. 295, de 10 de diciembre de 2013.

García, J. A. (2019). Psicología del Desarrollo I. Madrid: UNED.

Gimeno, J. (1988). El currículum: una reflexión sobre la práctica. Madrid: Morata.

González, M. A. y Jiménez, C. (2011). Pedagogía diferencial y atención a la diversidad. Madrid: Ramón Areces.

Gutiérrez, F. y Vila, J. O. (2011). Psicología del Desarrollo II. Madrid: UNED.

Jiménez, C. y González, M. A. (Coords.) (2011). Pedagogía diferencial y atención a la diversidad. Madrid: UNED.

Martínez, C. (2017). Evaluación de programas. Madrid: UNED.

Perrenoud, P. (2004). Desarrollar la práctica reflexiva en el oficio de enseñar. Barcelona: Graó.

Real Decreto 1105/2014, de 26 de diciembre, por el que se establece el currículo básico de la educación Secundaria Obligatoria y del Bachillerato. Boletín Oficial del Estado, núm. 3, de 3 de enero de 2015.

Salvador, F.; Rodríguez, J. L. y Bolívar, A. (2004). Diccionario Enciclopédico de Didáctica. Vol. II. Málaga: Aljibe.

Soler, E. (1995). Control de calidad e innovación. En XI Congreso Nacional de Pedagogía. Madrid: Bordón. Sociedad Española de Pedagogía.

Woolfolk, A. (2014). Psicología educativa. Madrid: Pearson. 
